\title{
The relationship between weight status and psychopathology in adolescence: The role of social support
}

Journal of Health Psychology

$0(0) \mathrm{I}-\mathrm{II}$

(C) The Author(s) 2013

Reprints and permissions:

sagepub.co.uk/journalsPermissions.nav DOI: 10.1 I77/|359/053/3482/62 hpq.sagepub.com

@SAGE

\author{
Marta A Freitas-Rosa', Sónia Gonçalves' and \\ Henedina Antunes 1,2,3 [AQ: 1]
}

\begin{abstract}
This study evaluated the differences between normal-weight and overweight adolescents in psychopathology, social support and emotional competences, and the role of social support in the relationship between psychopathology and weight. We examined a clinical group of 83 overweight/obese adolescents, a community group of 82 overweight/obese adolescents, and 205 adolescents with normal weight. No differences were found in psychopathology and emotional skills. Compared to healthy peers, however, the overweight/obese community group was less satisfied with their friendships, and the clinical group was less satisfied with their intimate support and social activities. Social support mediates the relationship between weight and psychopathology.
\end{abstract}

\section{Keywords}

adolescence, body mass index, mediator, psychological distress, social support[AQ: 4]

Excess weight and obesity are a growing global concern because of the adverse health and psychosocial consequences associated with these conditions in youths (Adair, 2008). Numerous studies have examined the relationship between obesity and psychopathology, but the results are not consistent (e.g. Brewis, 2003; Erermis et al., 2004; Zeller and Modi, 2006).

Although some investigators have found modest associations between psychopathology and obesity (Erermis et al., 2004; Franko et al., 2005), other studies have not observed such an association (Goodman and Whitaker, 2002). [AQ: 5]In contrast, additional studies primarily using clinical samples have found that psychopathological symptoms were inversely associated with obesity in adolescence (Zeller and Modi, 2006). A few studies have demonstrated modest differences in additional global psychological concerns (e.g. emotional wellbeing and peer concern) between overweight

\footnotetext{
University of Minho, Portugal[AQ: 2]

${ }^{2}$ Braga Hospital, Portugal

3ICVS/3B's-PT Government Associate Laboratory,

Portugal

\section{Corresponding author:}

Marta A Freitas-Rosa, School of Psychology, University of Minho, Campus Gualtar 47I 0 Braga, Portugal.

Email: martafreitasrosa@gmail.com[AQ: 3]
} 
and normal-weight adolescents (Falkner, 2001; Mond et al., 2011).[AQ: 6]

Longitudinal studies have shown that obesity predicts the emergence of psychiatric symptoms (e.g. Anderson et al., 2007) and that psychiatric symptoms predict obesity (e.g. Franko et al., 2005). Although several studies have documented the association between psychopathology and obesity in adolescence, there is inconsistency regarding the extent of those clinical problems. The relationship between obesity and psychological problems is not well established, and when the effects are observed, they typically are not clinically significant (e.g. Mond et al., 2011). Consequently, attention was turned to variables that might mediate this relationship, which explains why psychopathology impairs some obese individuals but not others (Flodmark, 2005). This diversity of results can also be explained by the presence of vulnerable subgroups across different samples, namely, by being exposed to adverse life conditions. Thus, a positive aspect of this study is its inclusion of three groups, to understand the differences between normal-weight adolescents, overweight/obese adolescents who have received treatment regarding weight loss, and overweight/obese adolescents who have not been treated for weight loss. Given the disparity of results found in the relationship between weight status and psychosocial dimensions, this study not only evaluates the association of obesity and such variables but also analyzes how social support mediates this complex relationship. Support from family and friends may help decrease the negative impact that an overweight adolescent's appearance has on his sense of self regardless of how others view the adolescent; however, this area is unexplored.

Parents and peers are important sources of social influence and may play an important role in the development of psychological problems associated with weight. The influence of teasing and stigmatizing experiences are notable in recent studies. Participants who did not show low global self-esteem reported relatively little weight-related teasing (Young-Hyman et al.,
2003). Furthermore, the history of weight-based stigmatization among participants in obesity treatment was associated with poorer body image and increased fear of fat (Latner et al., 2009). The social pressure to be thin that arises from family, friends, and society might also affect the relationship between increased adiposity, body dissatisfaction, and depression (Stice et al., 2003).

With these considerations in mind, we hypothesized that social support can mediate the relationship between body mass index (BMI) and psychopathological symptoms and that overweight/obese adolescents in treatment would score worse on psychological, social, and emotional variables, compared with overweight/obese and healthy weight peers from the community.

\section{Methodology[AQ: 7]}

\section{Participants}

Participants included 370 adolescents of both genders $(59.73 \%$ female), who were aged between 14 and 19 years in the 9th-12th grades from public schools located in the North of Portugal. The following describes each group: (a) the normal BMI community group: 205 adolescents with healthy weights (percentiles between P5 and P85), (b) the overweight/obese community group: 82 overweight/obese adolescents (weight percentiles $\geq$ P85) who were not receiving treatment for weight control, and (c) the overweight/obese clinical group: 83 overweight/obese adolescents (weight percentiles $\geq$ P85) who were receiving treatment for weight loss on the Pediatric Nutrition Outpatient Unit of Braga Hospital.

\section{Measures}

Weight status. The weights and heights of participants were measured after completing the questionnaires. To avoid weight-related constraints, each participant's weight was measured by the investigator, in private, standing 
and without shoes, looking forward, and positioned in the center of the scale. Participants' heights were measured with feet together and knees against a wall. Nurses performed the anthropometric evaluation at the hospital before the individual administration of the questionnaires. The BMIs were computed using the standard formula: weight $(\mathrm{kg}) /$ height $\left(\mathrm{m}^{2}\right)$. BMI percentiles were calculated using recent growth charts from the Centers for Disease Control and Prevention (Kuczmarski et al., 2000) and were then classified as having a normal weight or as overweight/obese based on the recent age- and sex-specific definitions of Cole et al. (2000).

Sociodemographic questionnaire. Basic demographic information was collected (age, school year, gender, parents' occupation, and residential area). The five socioeconomic levels (low, middle-low, middle, middle-high, and high) correspond to the five degrees of Graffar International Classification regarding occupation of the father.

Brief Symptom Inventory. The Brief Symptom Inventory (BSI; Derogatis, 1982; Portuguese version by Canavarro, 1999) is an abbreviated form of the Symptom Checklist-90. [AQ: 8]It consists of 53 Likert items and is used to identify clinically relevant psychological symptoms as well as their intensity (from 0 to 4 ) at a specific time point. Only the global severity index (GSI; the mean of the 53 symptoms) was used in the analyses of this study; this global index measures the current or past symptomatology level (in the last 2 weeks), and higher scores indicate more psychopathological symptoms. The psychometric characteristics of the Portuguese version attest to its satisfactory internal consistency (Canavarro, 1999), which justifies its use to identify psychological disturbances. The BSI has been shown to be a reliable and valid measurement of Portuguese adolescents. Specifically, regarding the GSI, our sample obtained a high level of internal consistency $(\alpha=.96)$.
Social Support Satisfaction Scale (Pais-Ribeiro, 1999). This self-report questionnaire consists of 15 items/statements, and its scale yields a total score of $1-5$ in which $1=$ "totally agree" and $5=$ "totally disagree." The Social Support Satisfaction Scale (SSSS) has a total index (with a maximum value of 75) and four subscales: satisfaction with friendships, satisfaction with intimate socioemotional support, satisfaction with family, and satisfaction with social activities. Greater scores represent more satisfaction with social support. The internal consistency in the current sample as reflected by Cronbach's $\alpha$ was $.83, .83, .70$, and .81 regarding satisfaction with friendships, satisfaction with intimacy, satisfaction with family, and satisfaction with social activities, respectively, and .83 for the total score. Thus, only the subscale satisfaction with social activities revealed a less reasonable internal consistency.

Emotional Skills and Competence Questionnaire. The Emotional Skills and Competence Questionnaire (ESCQ; Taksic, 2000; Portuguese version by Faria and Lima Santos, 2001) contains 45 Likert-type items ranging from $1=$ "never" to 6 = "always," in which greater scores reflect superior emotional skills. [AQ: 9]It has three subscales: (a) ability to perceive/understand emotion, (b) ability to express/label emotion, and (c) ability to manage and regulate emotion. The Cronbach's coefficient $\alpha$ in the current sample was .77 for the emotional perception subscale, .89 for the emotional expression subscale, and .82 for the emotional regulation subscale, showing a good internal consistency.

\section{Procedure}

In this study, we respected the following guidelines: the participants were excluded if they had neurological problems (e.g. epilepsy, meningitis, or mental retardation). We also excluded students who were following a program for weight control from the two community samples to ensure three well-defined groups. The 
existence of a control group with normal BMI was due to the need to examine whether the results were better explained by weight or other typical stressful factors of a specific stage of life like adolescence. It was decided to exclude participants with BMIs below the 5th percentile in this group to not bias the sample with possible cases of eating disorders such as anorexia.

After getting permission from hospitals and three academic institutions, informed consent was obtained from the parents and participants. Participation was voluntary, and the confidentiality of the data was ensured. At the schools, the classes were randomly selected by teachers. The investigator was always present to answer questions during the instrument administration. All the participants of the clinical group were randomly recruited by the pediatrician of the Pediatric Nutrition Outpatient Unit of Braga Hospital. At the evaluation moment, all the participants had already attended at least two treatment sessions. This study followed the ethical procedures outlined in the Declaration of Helsinki and was approved by the Ethics Committee of Braga Hospital.

\section{Data analyses}

All analyses were performed using SPSS statistical software (version 19.0). The significance level was set at $p<.05$, and values from .05 to .10 were defined as a trend. We began with an exploratory data analysis to test the assumptions of parametric tests that were present in the majority of cases. In cases where normality was not assumed, we computed both the parametric and the equivalent nonparametric tests, as suggested by Fife-Schaw (2006). We highlight the cases in the text in which both tests were applied; however, the results of both tests arrived at the same conclusions in general. Thus, we present only the parametric tests' results, because they are more robust and allow us to use multivariate analyses, while reducing both the number of tests conducted and the probability of the type I error.
Because our hypotheses were driven by prior findings of strong differences between clinical and community groups, the initial analyses were stratified by the criteria of receiving/not receiving weight-related treatments. Descriptive statistics were calculated for the participants' demographic characteristics. Pearson's rho $(r)$ assessed the correlation between the BMI, BSI, SSSS, and ESCQ scores. Analyses of variance (ANOVAs), multivariate analyses of variance (MANOVAs), and chi-square tests determined between-group differences. A linear multiple regression examined the variables associated with psychopathology. Multiple regression analyses tested the mediational role of social support variables, using the methods suggested by Baron and Kenny (1986). Psychopathology level served as the dependent variable (DV), and BMI was the independent variable (IV). Using MedGraph, a program to graphically depict mediation among three variables (the internet version 2.0) (Jose, 2003), we also calculated Sobel's $t$ (or $z$ ) value. This result must be sufficiently large, yielding a $p$ value of less than .05 , in order for significant mediation to be identified. In the regression analyses, BMI raw values were used, whereas for the betweengroup comparisons, sex- and age-specific BMI percentiles were considered.

\section{Results}

\section{Participant characteristics}

The mean ages of the control, the overweight/ obese community, and the overweight/obese clinical groups were 16.20 years (standard deviation $(S D)=1.19$ years), 16.29 years $(S D=1.57$ years), and 15.55 years $(S D=1.30$ years $)$, respectively. We found significant betweengroup differences in mean age $(p<.001)$; post hoc analyses revealed that the overweight/ obese clinical group was significantly younger than the overweight/obese community group ( $p$ $<.01)$ and the control group $(p<.001)$. The community groups did not differ with regard to age $(p=.914)$. 
Table I. MANOVA and ANOVA results regarding the psychosocial dimensions of three groups.

\begin{tabular}{|c|c|c|c|c|c|}
\hline & \multicolumn{2}{|l|}{ Community sample } & \multirow{2}{*}{$\begin{array}{l}\text { Clinical sample } \\
\text { Overweight } \\
(n=83), M(S D)\end{array}$} & \multirow[t]{2}{*}{$F$} & \multirow[t]{2}{*}{$p$} \\
\hline & $\begin{array}{l}\text { Normal BMI } \\
(n=205), M(S D)\end{array}$ & $\begin{array}{l}\text { Overweight } \\
(n=82), M(S D)\end{array}$ & & & \\
\hline \multicolumn{6}{|l|}{ Total scales } \\
\hline Total psychopathology (BSI) & $0.75(0.48)$ & $0.78(0.59)$ & $0.81(0.65)$ & 0.45 & .64 \\
\hline Total social support (SS) & $58.68(9.05)$ & $55.24(10.08)$ & $55.84(10.4)$ & 4.96 & $.007^{* * * *}$ \\
\hline \multicolumn{6}{|l|}{ Subscales } \\
\hline $\begin{array}{l}\text { Satisfaction with } \\
\text { friendships (SFr) }\end{array}$ & $4.27(0.7 \mathrm{I})$ & $3.92(0.89)$ & $4.09(0.83)$ & 6.05 & $.003^{* * * *}$ \\
\hline Satisfaction with intimacy (SI) & $3.83(0.89)$ & $3.62(0.99)$ & $3.53(1.00)$ & 2.95 & $.037^{*}$ \\
\hline Satisfaction with family (SFa) & $4.16(0.86)$ & $4.01(0.98)$ & $4.24(0.91)$ & 1.47 & .232 \\
\hline $\begin{array}{l}\text { Satisfaction with social } \\
\text { activities (SSA) }\end{array}$ & $3.17(1.00)$ & $3.03(0.92)$ & $2.81(0.99)$ & 3.86 & $.022 *$ \\
\hline \multicolumn{6}{|l|}{ Subscales } \\
\hline $\begin{array}{l}\text { Perceiving/understanding } \\
\text { emotion (PU) }\end{array}$ & $4.5 I(0.55)$ & $4.46(0.63)$ & $4.48(0.53)$ & 0.299 & .742 \\
\hline $\begin{array}{l}\text { Expressing and labeling } \\
\text { emotion (EL) }\end{array}$ & $4.5 I(0.70)$ & $4.46(0.76)$ & $4.47(0.7 \mathrm{I})$ & 0.213 & .808 \\
\hline $\begin{array}{l}\text { Managing and regulating } \\
\text { emotion (MR) }\end{array}$ & $4.65(0.57)$ & $4.59(0.59)$ & $4.58(0.61)$ & 0.705 & .495 \\
\hline
\end{tabular}

MANOVA: multivariate analysis of variance; ANOVA: analysis of variance; BMI: body mass index; BSI: Brief Symptom Inventory; SD: standard deviation.

$*_{p}<.05 ; * *_{p}<.01 ; * * * p<.001$.

The mean BMIs of the overweight/obese clinical, overweight/obese community, and control groups were $30.63 \mathrm{~kg} / \mathrm{m}^{2}\left(S D=3.95 \mathrm{~kg} / \mathrm{m}^{2}\right)$, $29.49 \mathrm{~kg} / \mathrm{m}^{2}\left(S D=4.19 \mathrm{~kg} / \mathrm{m}^{2}\right)$, and $20.79 \mathrm{~kg} / \mathrm{m}^{2}$ $\left(S D=1.94 \mathrm{~kg} / \mathrm{m}^{2}\right)$, respectively. As expected, we found significant differences in the mean BMI among the three groups $(p<.001)$; post hoc analyses revealed a higher average BMI for the groups of overweight/obese adolescents, compared with their healthy peers, and a marginally significant difference between the clinical and the overweight/obese community groups ( $p=.06)$ because the clinical group had a higher average BMI, compared with their overweight/ obese peers. It is common for obese/overweight communities to have lower BMIs than clinical groups (e.g. Erermis et al., 2004). This study also did not find significant between-group differences with regard to $\operatorname{sex}(p=.130)$ or socioeconomic status $(p=.323)$. The majority of all participants were from a middle-low socioeconomic level in the three groups $(61 \%$, $70.7 \%$, and $72.3 \%$ of the normal BMI group, overweight/obese community group, and overweight/obese clinical group, respectively).

\section{Between-group differences with regard to psychosocial dimensions}

Table 1 shows the mean and $S D$ of the psychosocial scales for all groups. No significant multivariate between-group differences were found with regard to psychopathology, either for BSI total score $(F(2,367)=0.45, p=.64)$ or its subscales (Wilks' $\lambda=.95, F(18,718)=1.02, p=$ $.43)$. We obtained nonclinical values (below a cutoff score of 1.7) for all groups. The overweight/obese groups tended to have higher indices of psychopathology (near the cutoff point) compared with normal-weight participants, although these differences did not reach statistical significance. 
There were also no significant multivariate between-group differences for all emotional dimensions evaluated (Wilks' $\lambda=.996, F(6$, $726)=0.26, p=.95)$. Similarly, univariate analyses did not reveal significant between-group differences in the ability to perceive/understand emotion $(F(2,365)=0.299, p=.742)$, ability to express/label emotion $(F(2,365)=0.213, p=$ $.808)$, or ability to manage/regulate emotion $(F(2,365)=0.705, p=.495)$.

However, significant differences were found not only for the total index of social support $(F(2,367)=4.96, p<.01)$ but also for the subscales (Wilks' $\lambda=.94, F(3,726)=2.86, p<$ $.01)$. Univariate tests revealed significant between-group differences regarding satisfaction with friendships $(F(2,367)=6.05, p<.01)$, and Gabriel's post hoc test showed that there were overweight/obese adolescents from the community who reported less satisfaction with friendships than adolescents with normal BMIs. However, the clinical group was less satisfied with their intimate support $(F(2,367)=2.95, p$ $<.05)$ and with their social activities, compared with the normal BMI group $(F(2,367)=3.86, p$ $<.05)$. No differences were found regarding satisfaction with family $(F(2,367)=1.47, p=$ .232; see Table 1).

\section{Associations between $\mathrm{BMI}$ and psychosocial dimensions}

Pearson's correlation $r$ was calculated for the associations between variables in the total sample. As expected, there was a high correlation between most variables. The three subscales of emotional competences were positively intercorrelated $(p<.001)$, as the four subscales and the total score of social support were also positively intercorrelated $(p<.001)$.

BMI was positively correlated with psychopathology $(p<.05)$ and negatively correlated with social support from friendships, intimate relationships, and social activities $(p<.01)$. Higher BMIs were associated with higher levels of psychopathology (total score) and with less satisfaction with social support. No emotional variables were associated with BMI. In contrast, psychopathology was significantly negatively correlated with all other social and emotional dimensions $(p<.001)$, except perceiving and understanding emotion.

\section{The mediating role of social support in the relationship between $\mathrm{BMI}$ and psychopathology}

A mediational model tested the unidirectional pathways of psychopathological symptoms, BMI, and social support. Specifically, BMI was set to predict psychopathology, and social support mediated this relationship. Based on the criteria outlined by Baron and Kenny (1986), mediation was confirmed because (a) social support is predicted by BMI $(\beta=-.20$, standard error $(S E)=.09, p<.001)$, (b) psychopathology is predicted by social support independent of BMI $(\beta=-.54, S E=.002, p<.001)$, and (c) the relationship between BMI and psychopathology is reduced or eliminated when social support is included in the model $(\beta=.02, S E=.004, p=$ .735). Perfect mediation is achieved if the IV no longer has an effect on the DV in the third equation (Baron and Kenny, 1986). [AQ: 10]As expected, mediation was achieved, and the complete mediation occurred, since the $\beta$ for the basic relationship between BMI and psychopathology (IV and DV) became nonsignificant, when the mediator was entered into the model. Sobel's method also confirmed a perfect (not partial) mediation $(z$ value $=2.22, p=.023$ ).

As an additional precaution, Baron and Kenny (1986) suggested that the mediator and the DV should be interchangeable, and if the DV acts as a mediator between the other two variables, then the model may be less reliable. In this study, this relationship was assessed, and it was found that the relationship between social support and BMI remained significant regardless of psychopathology. A preliminary analysis confirmed that BMI was related strongly to social support $(\beta=-.20, S E=.09$, $p<.001)$. 
The results indicated that social support had a mediating effect on the relationship between BMI and psychopathology. When the BMI and the total scores of social support and psychopathology were entered into the model, the results also revealed that this final model explained 29.5 percent of the variance of psychopathological symptoms among adolescents (adjusted $R^{2}=.29$, $F(2,367)=76.78, p<.001)$. When the different sources of support were analyzed separately, mediation was achieved on all subscales except for the family support. We observed that satisfaction with intimacy and friendships explained a larger percentage of the variance in the regression model $(20.3 \%$ each), compared with satisfaction with social activities $(10.2 \%)$. The adjusted $R^{2}$ and the $F$-test statistics for the final model, including the mediators, were as follows for each perceived source of support: satisfaction with friendships - adjusted $R^{2}=.20, F(2,367)=$ $46.89, p<.001$; satisfaction with intimacyadjusted $R^{2}=.20, F(2,367)=47.87, p<.001$; and satisfaction with social activities - adjusted $R^{2}=.10, F(2,367)=21.94, p<.001$.

\section{Discussion}

This study explored the differences among normal-weight adolescents versus those from the community who are overweight/obese versus those in treatment who are overweight/obese, with regard to psychopathology, emotional competences, and social support. Moreover, we examined the role of social support on the relationship between psychopathology and weight in adolescence. Interestingly, we found that the rates of psychopathology in the overweight/obese group in treatment are similar to those of the overweight/obese community group and normalweight adolescents. Thus, in our study, psychopathology does not differentiate overweight adolescents who seek treatment from those in the community. However, Braet et al. (1996) hypothesized that parents of adolescents who seek treatment could be more pathological than parents not referring their children to a clinic. As a result of their own problems, they can be more responsive to information about therapeutic services regarding children problems. [AQ: 11]Another possibility for parents to take their children to the pediatric outpatient clinic may be their belief that seeking clinical help can be a way to cope with any of their children's psychosocial problems (Braet et al., 1996).

The finding that both community samples of overweight/obese and nonoverweight adolescents are equally in terms of psychological adjustment is consistent with recent community-based studies, which suggest that overweight/obese children and adolescents in the general population are no more likely to suffer from psychological problems than their normal-weight counterparts (Brewis, 2003; Lamertz et al., 2002).

In addition, it is noteworthy that the majority of adolescents recruited from the outpatient population were in the middle of treatment. Obviously, health professionals are able to increase the self-confidence of patients and their psychological well-being. If the clinical sample recruitment had been conducted during the first visit, without possible positive effects of the medical intervention, then the results might be more consistent with the recent literature, which suggests that there is poorer psychological well-being in clinical samples compared with community samples (e.g. Erermis et al., 2004; Vila et al., 2004). Although this did not occur in our study, our results enhance the positive impact that a continuing outpatient weight reduction treatment may have for overweight/obese outpatients, not only regarding weight loss but also regarding their psychological functioning and quality of life, as shown in the report of obese adult outpatients from a study of Kolotkin et al. (2004).

There were no significant between-group differences concerning emotional competences. These results are in line with Neumark-Sztainer et al. (1997), who showed that young people had not distinguished themselves using psychosocial variables, such as emotional well-being, when grouped by weight. Mond et al. (2011) recently compared obese and normal-weight 
peers and obtained similar results. Although they found that obese adolescents tend to have lower self-esteem and higher depressive moods than normal-weight participants, the differences were not statistically significant. Thus, in the absence of other mediating variables such as body and shape dissatisfaction, excess weight may be associated with little or no impairment in mental health (Mond et al., 2011).

Regarding social support, we found that the clinical group had less satisfaction with intimacy and social activities than normal BMI group. Moreover, we found that the overweight/obese community group had less satisfaction with their friendships than their normal-weight peers. These results are consistent with Falkner (2001), who found that overweight teens were less likely to spend time with friends over the last week than their counterparts. [AQ: 12]Weight stigmatization can also be the reason why these adolescents are less satisfied with social support. Obesity stigmatization is particularly concerning during adolescence when the formation of social relationships is salient (Puhl and Latner, 2007). Many studies have shown positive associations between overweight and overt/relational victimization in youths (e.g. Janssen et al., 2004; Neumark-Sztainer et al., 2002; Pearce et al., 2002). A few recent results are consistent with our data and suggest that obese and overweight adolescents are at greater risk of mistreatment and bullying behaviors by peers and may have fewer opportunities to develop intimate relationships (Janssen et al., 2004; Pearce et al., 2002). In addition, another study found that students with strong weight-based stereotypes reported less willingness to engage in social, academic, or recreational activities with overweight peers compared with thin peers (Greenleaf et al., 2006). These forms of victimization may contribute to isolation increasing the negative social effects of their weight problems.

Furthermore, we found greater associations between psychopathological, emotional, social, and weight dimensions, while, in addition, Zeller and Modi (2006) found robust associations among impaired health-related quality of life, depressive symptoms, lower perceived social support, and degree of obesity. Vander Wal (2012) also found that a low life satisfaction, high negative affect, and body size dissatisfaction were associated with unhealthy weight control behaviors among adolescents. As expected, our analyses revealed that high social support was inversely associated with psychopathology. Higher BMI is associated with increases in psychopathological symptoms, and this effect is strongly mediated by lower satisfaction with intimacy, friendships, and social activities. Our mediational model is consistent with the results found by Santoncini et al. (2012), who found that disordered eating behaviors did not interact significantly with BMI but were linked with criticism of overweight/obese teenage girls. These results are consistent with existent literature, which suggests that increases in BMI prevent adolescents from participating in the same activities as their peers, not only because they are incapable of performing some activities but also because they are frequently teased (Puhl and Latner, 2007). Overweight/obese adolescents tend to isolate themselves to avoid such humiliations; however, when they are away from their peers, they miss experiences that are associated with mental and healthy growth (Puhl and Latner, 2007). Similarly, Wit et al. (2010) found that the association between major depression and obesity was partly influenced by the few social activities in which these individuals were involved. Davison and Birch (2002) also noted that peer teasing mediated the associations between weight and self-concept. Similarly, our study revealed that lower satisfaction with social support was associated with psychopathology, which eliminated the contribution of weight. This finding is consistent with Xie et al. (2005), who found that the effect of perceived peer isolation on the relationship between BMI and depressive symptoms among adolescents was sustained only by the low perceived availability of social support.

This study has strengths including its reliance on objective measures of height and 
weight. In turn, the division of overweight/obese adolescents in clinical and community groups is a major advantage of this study because recent researches show different levels of psychopathology in clinical and community samples (e.g. Braet et al., 1997; Erermis et al., 2004; Pierce and Wardle, 1997). This study is the first testing the role of social support as a mediator of the relationship between BMI and psychopathology. Our findings are innovative because they illustrate the importance of support provided by significant others in explaining the links between weight and psychopathology.

In contrast, there are also several limitations regarding the findings of this study. First, it relies on self-reports, and second, its cross-sectional design precludes conjecture regarding causality, direction of effect, and the temporal sequence of events. Longitudinal and qualitative studies might also provide information regarding the development of psychological disturbances and its relationship with weight, if there are bidirectional effects. Another limitation was the option of not analyzing data dividing the groups by gender and by overweight/ obesity status. Although several differences are known in the literature regarding gender (Smolak, 2004) and overweight/obesity status (Falkner et al., 2001) with girls and obese youths reporting more adverse social and psychological correlates, we decided to give priority to the division of receiving/not receiving treatment. If we subdivided the groups by gender and overweight/obesity status, we would compromise statistical power and deviate from our goal of explaining results by clinical status.

This study provides evidence that being overweight is not necessarily related to the development of psychopathology. Our results lend increasing support to a mediational model, in which social support affects the relationship between BMI and psychopathology.

In addition, body image also may have an impact in psychological well-being, with females less satisfied with their body than males (McCabe et al., 2012). [AQ: 13]Thus, it would be important to explore recent data (Vander
Wal, 2012) that point to the mediational role of body satisfaction in the relationship between BMI and the existence of psychopathology and unhealthy weight control strategies.

Our study has some important practical implications suggesting that intervening at the level of social support may protect overweight adolescents from developing poor mental health. Based on a recent review (Bogle and Sykes, 2011), there is evidence that parents are the main agents for treating childhood obesity. While dealing with overweight adolescents, and according to our study, the peers and intimate partners may also have a large impact in their psychological well-being and, consequently, in their weight loss. Because the vast majority of children attend school, Swiss et al. (2010) enhance the joint efforts of school stakeholders in preventing obesity and encouraging healthy behaviors and reinforcing peer and parental efforts through positive health messages. For example, health and school professionals might create programs to promote social competences in overweight adolescents and educate children on the consequences of peer discrimination.

In addition, exercise interventions can also provide benefits in well-being and self-confidence of obese adolescents (Daley et al., 2008). Nevertheless, as suggested by other studies (e.g. Blaine et al., 2007; Campbell and Hausenblas, 2009; Wilson et al., 2012; Wright et al., 2012), weight and exercise interventions may increase their chances of success if it is done along with the social support improvement and groupmediated cognitive-behavioral strategies, promoting positive changes in self-esteem, body image, and psychopathology.

\section{Funding}

This research received no specific grant from any funding agency in the public, commercial, or not-forprofit sectors.[AQ: 14]

\section{References}

Adair LS (2008) Child and adolescent obesity: Epidemiology and developmental perspectives. Physiology \& Behavior 94: 8-16. 
Anderson SE, Cohen P, Naumova EN, et al. (2007) Adolescent obesity and risk for subsequent major depressive disorder and anxiety disorder: Prospective evidence. Psychosomatic Medicine 69: 740-747.

Baron R and Kenny D (1986) The moderator-mediator variable distinction in social psychological research: Conceptual, strategic, and statistical considerations. Journal of Personality and Social Psychology 51: 1173-1182.

Blaine BE, Rodman J and Newman JM (2007) Weight loss treatment and psychological wellbeing: A review and meta-analysis. Journal of Health Psychology 12(1): 66-82.

Bogle V and Sykes C (2011) Psychological interventions in the treatment of childhood obesity: What we know and need to find out. Journal of Health Psychology 16(7): 997-1014.

Braet C, Mervielde I and Vandereycken W (1997) Psychological aspects of childhood obesity: A controlled study in a clinical and nonclinical sample. Journal of Pediatric Psychology 22(1): 59-71.

Brewis A (2003) Biocultural aspects of obesity in young Mexican schoolchildren. American Journal of Human Biology 15(3): 446-460.

Campbell A and Hausenblas HA (2009) Effects of exercise interventions on body image: A meta-analysis. Journal of Health Psychology 14(6): 780-793.

Canavarro MC (1999) Inventário de Sintomas Psicopatológicos-B.S.I. In: Simões M, Gonçalves $\mathrm{M}$ and Almeida L (eds) Testes e Provas Psicológicas em Portugal, vol. 2. Braga: APPORT/ SHO.[AQ: 15]

Cole TJ, Bellizzi MC, Flegal KM, et al. (2000) Establishing a standard definition for child overweight and obesity: International survey. British Medical Journal 320: 1-6.

Daley AJ, Copeland RJ, Wright NP, et al. (2008) "I can actually exercise if I want to; It isn't as hard as I thought": A qualitative study of the experiences and views of obese adolescents participating in an exercise therapy intervention. Journal of Health Psychology 13(6): 810-819.

Davison KK and Birch LL (2002) Processes linking weight status and self-concept among girls from ages 5 to 7 years. Developmental Psychology 38(5): 735-748.

Erermis S, Cetin N, Tamar M, et al. (2004) Is obesity a risk factor for psychopathology among adolescents? Pediatrics International 46(3): 296-301.
Falkner NH, Neumark-Sztainer D, Story M, et al. (2001) Social, educational, and psychological correlates of weight status in adolescents. Obesity Research 9(1): 32-42.

Faria L and Lima Santos N (2006) Competência emocional: Adaptação e validação intercultural do Emotional Skills and Competence Questionnaire (ESCQ). In: Machado C, Almeida L, Guisande MA, et al. (Coords)Actas da XI Conferência Internacional de Avaliação Psicológica: Formas e Contextos. Braga: Psiquilíbrios Edições, pp. 349-356.

Fife-Schaw C (2006) Levels of measurement. In: Breakwell GM, Hammond S, Fife-Schaw C, et al. (eds) Research Methods in Psychology (3rd edn). London: SAGE.[AQ: 16]

Flodmark C-E (2005) The happy obese child. International Journal of Obesity 29: S31-S33.

Franko DL, Striegel-Moore RH, Thompson D, et al. (2005) Does adolescent depression predict obesity in Black and White young adult women? Psychological Medicine 35: 1505-1513.

Goodman E and Whitaker R (2002) A prospective study of the role of depression in the development and persistence of adolescent obesity. Pediatrics 109(3): 497-504.

GreenleafC, Chambliss H, Rhea DJ, et al. (2006) Weight stereotypes and behavioral intentions toward thin and fat peers among White and Hispanic adolescents. Journal of Adolescent Health 39: 546-552.

Janssen I, Craig WM, Boyce WF, et al. (2004) Associations between overweight and obesity with bullying behaviors in school-aged children. Pediatrics 113(5): 1187-1194.

Jose PE (2003) MedGraph-I: A programme to graphically depict mediation among three variables: The Internet version, version 2.0. Victoria University of Wellington, New Zealand. Available at: http://www.victoria.ac.nz/staff/paul-josefiles/medgraph/medgraph.php(accessed 2 March 2012).[AQ: 17]

Kolotkin RL, Crosby RD, Williams GR, et al. (2004) Health-related quality of life and weight loss. Obesity Research 9(9): 564-571.

Kuczmarski RJ, Ogden CL, Grummer-Strawn LM, et al. (2000) CDC Growth Charts: United States. Advance Data from Vital and Health Statistics (publication no. 314). Hyattsville, MD: National Center for Health Statistics.

Lamertz CM, Jacobi C, Yassouridis A, et al. (2002) Are obese adolescents and young adults at higher 
risk for mental disorders? A community survey. Obesity Research 10(11): 1152-1160.

Latner JD, Wilson GT, Jackson ML, et al. (2009) Greater history of weight-related stigmatizing experience is associated with greater weight loss in obesity treatment. Journal of Health Psychology 14(2): 190-199.

McCabe MP, Fuller-Tyszkiewicz M, Mellor D, et al. (2012) Body satisfaction among adolescents in eight different countries. Journal of Health Psychology 17(5): 693-701.

Mond J, van den Berg P, Boutelle K, et al. (2011) Obesity, body dissatisfaction, and emotional well-being in early and late adolescence: Findings from the project EAT study. Journal of Adolescent Health 48(4): 373-378.

Neumark-Sztainer D, Falkner N, Story M, et al. (2002) Weight-teasing among adolescents: Correlations with weight status and disordered eating behaviors. International Journal of Obesity 26: 123-131.

Neumark-Sztainer D, Story M, French S, et al. (1997) Psychosocial concerns and health-compromising behaviors among overweight and nonoverweight adolescents. Obesity Research 5: 237-249.

Pais-Ribeiro JL (1999) Escala de Satisfação com o Suporte Social (ESSS). Análise Psicológica 3(XVII): 547-558.

Pearce MJ, Boergers MJ and Prinstein MJ (2002) Adolescent obesity, overt and relational peer victimization, and romantic relationships. Obesity Research 10(5): 386-393.

Pierce JW and Wardle J (1997) Cause and effect beliefs and self-esteem of overweight children. Journal of Child Psychology and Psychiatry 38(6): 645-650.

Puhl RM and Latner JD (2007) Stigma, obesity and the health of the nation's children. Psychological Bulletin 133(4): 557-580.

Santoncini CU, Martin VM, Garcia FJ, et al. (2012) Disordered eating behaviors and body weight and shape relatives' criticism in overweight and obese 15- to 19- year-old females. Journal of Health Psychology. Available at: http://dx.doi. org/10.1177/1359105311435945

Smolak L (2004) Body image in children and adolescents: Where do we go from here? Body Image 1: $15-28$.

Stice E, Maxfield J and Wells T (2003) Adverse effects of social pressure to be thin on young women: An experimental investigation of the effects of "fat talk." International Journal of Eating Disorders 34: 108-117.

Swiss SB, Akré C and Suris J-C (2010) Obesity prevention opinions of school stakeholders: A qualitative study. Journal of School Health 80(5): 233-239.

Vander Wal JS (2012) Unhealthy weight control behaviors among adolescents. Journal of Health Psychology 17(1): 110-120.[AQ: 18]

Vila G, Zipper E, Dabbas M, et al. (2004) Mental disorders in obese children and adolescents. Psychosomatic Medicine 66(3): 387-394.

Wardle J, Volz C and Golding C (1995) Social variation in attitudes to obesity in children. International Journal of Obesity 19: 562-569.[AQ: 19]

Whaley AL, Smith M and Hancock A (2011) Ethnic/racial differences in the self-reported physical and mental health correlates of adolescents obesity. Journal of Health Psychology 7: 10481057.[AQ: 20]

Wilson AJ, Jung ME, Cramp A, et al. (2012) Effects of a group-based exercise and self-regulatory intervention on obese adolescents' physical activity, social cognitions, body composition and strength: A randomized feasibility study. Journal of Health Psychology 17(8): 1223-1237.[AQ: 21]

Wit LM, Fokkema M, van Straten A, et al. (2010) Depressive and anxiety disorders and the association with obesity, physical, and social activities. Depression and Anxiety 27(11): 1057-1065.

Wright F, Boyle S, Baxter K, et al. (2012) Understanding the relationship between weight loss, emotional well-being and health-related quality of life in patients attending a specialist obesity weight management service. Journal of Health Psychology 0(0): 1-13.[AQ: 22]

Xie B, Chou C-P, Spruijt-Metz D, et al. (2005) Effects of perceived peer isolation and social support availability on the relationship between body mass index and depressive symptoms. International Journal of Obesity 29: 1137-1143.

Young-Hyman D, Schlundt DG, Herman-Wenderoth L, et al. (2003) Obesity, appearance, and psychosocial adaptation in young African American children. Journal of Pediatric Psychology 28(7): 463-472.

Zeller MH and Modi AC (2006) Predictors of healthrelated quality of life in obese youth. Obesity 14(1): 122-130. 